\title{
ACCIONES DE WRONGFUL CONCEPTION EN CHILE: UNA PROPUESTA DE FUNDAMENTACIÓN
}

\author{
Hugo Cárdenas Villarreal ${ }^{1}$, José Antonio Sánchez Rubín ${ }^{1}$
}

Resumen: En el presente artículo, los autores tratan el tópico de admisibilidad en Chile de las acciones de responsabilidad civil de wrongful conception o anticoncepciones fallidas. Utilizando referencias hacia la práctica en el derecho comparado y al derecho internacional de los derechos humanos, los autores argumentan a favor de fundamentar su reparación en el derecho a la autodeterminación reproductiva de las mujeres.

Palabras clave: jurisprudencia médica, litigación, mal praxis, fertilización, pacientes, padres

\section{Wrongful conception claims in Chile: a proposal for their justification}

Abstract: In this article, the authors refer to the wrongful conception claims, particularly the controversy of their admission in Chile. On the basis of comparative and human rights international law, the authors argue in favor of the women's right to reproductive self-determination as the foundation of their compensation.

Key words: medical jurisprudence, litigation, malpractice, fertilization, patients, parents

\section{Açóes de wrongful conception no Chile: uma proposta de fundamentaçáo}

Resumo: Neste artigo, os autores abordam o tema da admissibilidade no Chile das açôes de responsabilidade civil de wrongful conception ou anticoncepçóes fracassadas. Com base em referências para a prática no direito comparado e no direito internacional dos direitos humanos, os autores argumentam a favor de fundamente sua reparaçáo o direito à autodeterminaçáa reprodutiva das mulheres.

Palavras-chave: jurisprudência médica, contencioso, má práxis, fertilização, pacientes, pais

\footnotetext{
${ }^{1}$ Facultad de Derecho, Universidad de Chile, Chile

Corespondencia: hcardenas@derecho.uchile.cl
} 


\section{Introducción}

En la reciente práctica judicial chilena se han empezado a presentar demandas de responsabilidad civil fundadas en lo que la literatura especializada ha venido denominando casos de wrongful conception, wrongful pregnancy o anticoncepciones fallidas $(1,2)$. Esta nueva categoría de casos comprende todas aquellas pretensiones indemnizatorias que se asocian con la ineficacia de un tratamiento anticonceptivo o de esterilización, que tiene como consecuencia el nacimiento de un niño.

Aunque en principio estos casos pueden parecer supuestos simples de negligencia médica, la solución de los mismos supone enfrentar, entre otros problemas, la admisibilidad de su reparación desde la perspectiva de la legitimidad del interés cuya vulneración se alega. En términos simples, la pregunta que los autores se plantean es si la vida, como tal, puede ser considerada como un daño que el Derecho debe reparar.

En este artículo se analizará este singular problema, que suele ir acompañado de otras dificultades técnicas, a partir de las diferentes respuestas que se han dado a las acciones de wrongful conception en Chile y en aquellos países donde el tema ha experimentado un mayor grado de desarrollo. Finalmente, a la luz de los antecedentes examinados, se propondrá una justificación para las indemnizaciones que pretenden reparar este daño, desde la perspectiva de los derechos reproductivos de la madre.

\section{Antecedentes y contexto de estas acciones}

Las primeras acciones por wrongful conception se presentaron en la segunda mitad del siglo que recién pasó, en países con ordenamientos jurídicos tan disímiles como los que tienen los Estados Unidos y Alemania. Desde entonces, el desarrollo de este tópico (en un contexto de consolidación de la autonomía reproductiva como derecho fundamental) ha ido caracterizando la categoría wrongful conception con rasgos distintivos a los que presenta un caso "simple" de negligencia médica.

Lo anterior se puede explicar porque, a diferen- cia de las personas que acuden al médico cuando tienen una enfermedad, las personas que deciden someterse a este tipo de tratamientos están guiadas por motivaciones que se relacionan con la intención de evitar gastos derivados del embarazo y la crianza que no están en condiciones económicas de asumir; la idea de prevenir la contingencia del nacimiento de un hijo atendiendo otras circunstancias personales (por ejemplo, una discapacidad visual o una edad riesgosamente avanzada) o, simplemente, con la decisión de no procrear de manera definitiva o en un momento determinado de su vida.

En Chile, el surgimiento de estos casos está determinado por la circunstancia de que la política pública de planificación familiar hizo que el número de usuarias de métodos anticonceptivos creciera en un 123\% entre 1990 y 2012 (600.374 mujeres en 1990 a 1.339.444 en 2012), aumentando consecuentemente las probabilidades de que se materialicen contingencias del tipo que se analizan(3); y por la circunstancia de que en los últimos años viene operando un cambio en la mentalidad de los pacientes chilenos, que los ha hecho mucho más decididos a interponer reclamos antes los tribunales de justicia(4). Como ha sido agudamente observado, estamos pasando de un paciente pasivo a uno más bien "impaciente" (5).

El problema de las anticoncepciones fallidas presenta importantísimos desafíos teóricos y prácticos que se vinculan con la determinación del vínculo causal entre el tratamiento médico y embarazo no deseado; la aceptación de la idea de que el nacimiento de un hijo pueda ser considerado un daño, y, en su caso, con los criterios que deberán tomarse en cuenta para evaluar las indemnizaciones que correspondan. Con todo, en este trabajo nos preocuparemos de la pregunta que aparece como más importante: ¿puede el nacimiento de un hijo dar lugar a un injusto cuyo resultado, el dańo, deba ser reparado?

\section{Las anticoncepciones fallidas y la responsabi- lidad civil médica}

La responsabilidad civil por anticoncepciones fallidas cubre supuestos de embarazo por las esterilizaciones negligentemente practicadas; por las 
esterilizaciones en las que no se informan oportunamente sus consecuencias; por tratamientos que suponen la colocación negligente de un mecanismo intrauterino, y por la circulación o comercialización de mecanismos anticonceptivos ineficaces(6).

Estos casos tienen como fundamento dos presupuestos conceptuales: la vinculación causal entre un fallo o defecto en el tratamiento de anticoncepción y el embarazo, y la circunstancia de que el hijo o hija nazca sano o sana(7). Esta última condición suele ser un criterio relevante para diferenciar los casos de wrongful conception de otros casos de wrongful birth y wrongful life, con los cuales guarda ciertas semejanzas. Estos últimos encuentran fundamento en el error de diagnóstico o la omisión negligente de informar a los padres de la existencia de una enfermedad (o grave defecto del feto), que impide el ejercicio del derecho de la madre a poner término al embarazo(8).

Sin embargo, a la luz de los casos que se observan en el derecho comparado, cabe matizar al menos en dos sentidos las diferencias entre los casos de wrongful conception y los de wrongful birth y wrongful life.

En primer lugar, porque en países como Alemania o Inglaterra los embarazos no deseados producto de abortos fallidos se comprenden como casos de wrongful conception(9). Y este matiz tiene relevancia en nuestro medio, porque la reciente promulgación de la ley que regula la despenalización de la interrupción voluntaria del embarazo en tres causales aumentaría considerablemente el catálogo de casos que cubriría esta categoría si se compartiera una comprensión semejante a aquella de los países mencionados. $\mathrm{Si}$, por ejemplo, pese a que la madre se sometió a un aborto (digamos, bajo la causal de violación que establece el número 3 del actual artículo 119 del Código Sanitario), el nacimiento se produce, ¿es, acaso, relevante esta razón para los efectos de admitir la demanda civil? En hipótesis distintas a esta, ¿podría admitirse la reparación por la anticoncepción fallida en este caso, pero negarla en la hipótesis de inviabilidad del feto? Si el fallo en el aborto significó el nacimiento del hijo pero la muerte de una madre cuya vida no estaba en peligro, ¿cómo habrá de valorarse en derecho ese resultado?
En segundo lugar, deben matizarse las diferencias porque el ámbito explicativo de la doctrina del wrongful conception parece ser más amplio que lo que los presupuestos identificados sugieren, pues el estado de salud del hijo inesperado no ha sido del todo irrelevante en la experiencia comparada. En efecto, la enfermedad o discapacidad pueden ser consideradas al momento de determinar el monto o quantum de la indemnización. Por vía de ejemplo, en el Reino Unido, en Parkinson v St. James and Seacroft University Hospital NHS Trust, la Court of Appeal concedió una indemnización por los costos extras que supone traer al mundo a un hijo discapacitado.

Desde el punto de vista jurídico, un daño es indemnizable cuando se concibe como la lesión de un interés o un derecho jurídicamente protegido. Por lo mismo, una dificultad técnica que se hace presente en estos casos está en determinar qué interés o derechos pueden ser lesionados con el nacimiento de una criatura inesperada.

Antes de proponer una explicación que pueda fundamentar la procedencia de estas acciones, conviene analizar las soluciones que se han dado, tanto en el Derecho comparado como en el Derecho chileno, al problema que hemos presentado.

\section{Las soluciones del Derecho comparado}

Ya se ha visto cómo el problema de las anticoncepciones fallidas ha sido abordado desde hace algunas décadas en el Derecho comparado, en el que se aprecia una firme tendencia hacia la aceptación de este tipo de acciones, aun cuando las diferencias entre las resoluciones judiciales son más bien numerosas. Así, por ejemplo, existen países en los que los tribunales de justicia sencillamente no admiten este tipo de acciones.

En el Derecho comparado, uno de los principales nudos de discusión gira en torno a la pregunta por la posibilidad de que una vida humana, sea cual sea el estado en el que sea alumbrada, pueda ser comprendida por el derecho como un "daño indemnizable".

Respecto de este punto, en 1991 la Corte de Casación francesa rechazó una acción de responsabilidad civil por wrongful conception, argu- 
mentando que la existencia de un hijo (en ese caso, sano) no da lugar, por sí misma, a un daño reparable(10). En España, en principio, la regla es similar, pues mediante sentencia de 4 de noviembre de 2008 el Tribunal Supremo afirmó que "no hay nacimientos equivocados o lesivos" y que, consecuentemente, "toda vida es digna de ser vivida" (11).

Sin embargo, aun en las jurisdicciones de los países referidos en el párrafo precedente se han admitido excepciones o atenuaciones a la regla de no reparación de este tipo de casos. Así, el propio Tribunal Supremo español, en un fallo de 2015 , determinó que, aunque en principio no hay nacimientos lesivos, sí hay derecho a una indemnización cuando ese nacimiento implica una vulneración del "poder de la persona a autodeterminarse" (12).

Curiosamente, a pesar de las reticencias observadas respecto de la admisibilidad de estas acciones, las cortes extranjeras suelen no tener inconvenientes para acoger la reparación de gastos asociados al embarazo, al parto del hijo o hija inesperados y, en algunos casos, a los daños morales generados por la negligencia de los profesionales. La normalidad de estos perjuicios, en tanto categorías largamente asentadas en esos ordenamientos, pareciera ser suficiente como para derrotar las reservas valorativas que generan estas acciones.

Por ejemplo, entre las jurisdicciones que tradicionalmente han concedido indemnizaciones por este tipo específico de daño se cuenta el Reino Unido. En el caso de referencia en la materia (McFarlane v Tayside Health Board 1999), se concedió una indemnización con el objeto de reparar el dolor y los malestares sufridos por el embarazo y parto de un hijo sano pero no deseado, así como de la pérdida de los ingresos durante el embarazo. Las cortes del Reino Unido, sin embargo, parecen reacias a conceder indemnizaciones por "gastos adicionales de crianza", aun cuando en Parkinson v St. James and Seacroft University Hospital NHS Trust de 2011 se concedió esta última partida indemnizatoria en atención a que el hijo o hija inesperado nació con una discapaci$\operatorname{dad}(13)$.
En el derecho estadounidense se encuentra uno de los ejemplos más interesantes en cuanto a la variedad de las respuestas que pueden darse en torno al problema que se analiza (lo que se explica por el sistema federal bajo el que su legislatura y judicatura se organizan). Allí se observan al menos dos formas de resolver este tipo de litigios: una mayoritaria, que solo repara los gastos en que efectivamente se incurrió con motivo del parto y el procedimiento de esterilización; y otra, minoritaria (Arizona, Connecticut y Massachusetts), que habilita una indemnización que incluye, además de las partidas indemnizatorias contempladas por la tendencia mayoritaria, los costos de crianza del hijo o hija inesperado. Adicionalmente, también se observa una variante en este último modelo de solución que pondera, al fijar la cuantía de la indemnización, los costos de manutención con los beneficios emocionales y financieros de tener un hijo(14).

\section{Las soluciones del Derecho chileno}

Entre los autores que han abordado el problema a la luz del ordenamiento jurídico chileno, ninguno se ha mostrado explícita y contundentemente a favor de que se indemnice este tipo de "daño" (7). Por el contrario, se encuentra alguna fuerte oposición. Así, por ejemplo, en opinión de Hernán Corral, "La tradición humanística ha siempre defendido que todo ser humano, cualquiera sea la forma en que llegue al mundo, es valioso objetiva e incondicionalmente $\mathrm{y}$, como tal, digno de tutela por parte del derecho. Por ello, parece contradictorio que pueda ser calificado jurídicamente como un daño susceptible de reparación. Lo digno y valioso en sí mismo (...) no puede ser a la vez perjudicial y nocivo" (15).

Por su parte, por regla general, la jurisprudencia chilena no ha explicitado razones de índole valorativa o filosófica que justifiquen el rechazo absoluto de estas acciones. Por el contrario, puede afirmarse que existe una tendencia hacia la reparación de los costos del embarazo y del parto, así como a la reparación del sufrimiento y detrimento psicológico de la madre bajo la categoría de dańo moral(16).

De momento, los costos de manutención o crianza del hijo inesperado no han sido considerados 
daños reparables. Así, por ejemplo, en Morales Castillo con Sociedad de Profesionales y Escudero Schiappacasse 2014 se negó la reparación de estos gastos. El juzgador entendió que lo que se estaba demandando eran "alimentos", y la obligación de alimentar es una obligación que no puede ser impuesta a un médico o sociedad de profesionales, en tanto que recae en los progenitores según las normas del Código Civil(17).

Como puede apreciarse, los jueces chilenos han empezado a conceder parcialmente indemnizaciones en caso de anticoncepciones fallidas, aunque los fallos existentes no han explicitado la razón por la que otorgan este tipo de indemnizaciones. La respuesta a esta cuestión importa porque aportará el fundamento de la responsabilidad de los profesionales y prestadores involucrados, y porque arrojará luz sobre la extensión de la reparación respecto de ítems indemnizatorios que se han presentado como conflictivos (p. e. los gastos de crianza del hijo o hija no esperados).

\section{La lesión al derecho a la autodeterminación del plan de vida}

En el ámbito del Derecho internacional de los derechos humanos pareciera existir consenso en cuanto al reconocimiento, directo e indirecto, de los derechos reproductivos y derechos sexuales. El punto de partida de su afirmación se encuentra en el artículo 16 de la Convención sobre la Eliminación de Todas las Formas de Discriminación Contra la Mujer de 1979 (CETFDCM, o más conocida como CEDAW, por su nombre en inglés), que establece la obligación de los Estados Parte de asegurar, en condiciones de igualdad entre hombres y mujeres, el derecho a decidir libre y responsablemente el número de sus hijos, el intervalo entre los nacimientos y el acceso a la información, educación y medios que les permitan ejercerlos. A este instrumento, se suman el Informe de la Conferencia Internacional sobre Población y Desarrollo (CIPD), celebrada en el Cairo entre el 5 y 13 de septiembre de 1994, y la Conferencia Mundial sobre la Mujer, celebrada entre el 4 y 15 de septiembre de 1995 en Beijing, que profundizan en torno a la libertad a planificar la paternidad y afirman el derecho de la mujer "a tener control sobre las cuestiones relativas a su sexualidad, incluida su salud sexual y reproductiva, y decidir libremente respecto a estas cuestiones"(18).

A partir de este contexto normativo, que incluye el derecho a la libertad reconocido en los artículos $3^{\circ}$ de la Declaración Universal de Derechos Humanos y $7^{\circ}$ de la Convención Americana de Derechos Humanos, se construye por vía interpretativa un haz de derechos fundamentales que, en su conjunto, conforman los derechos reproductivos, entre los cuales se encuentra el derecho a la autonomía reproductiva, cuyo contenido está conformado por la facultad de decidir el número e intervalo de hijos(18).

La lesión del plan de vida (autodeterminación) puede ser el fundamento inmediato de las acciones de wrongful conception en Chile, pues este interés puede reconducirse a la vulneración de garantías fundamentales consistentemente reconocidas, como lo son la dignidad humana y el libre desarrollo de la personalidad (artículo $1^{\circ}$ de la Constitución Política de la República).

\section{Primer reconocimiento jurisprudencial: $B a b a-$ mondes con Servicio de Salud de Chiloé 2016}

Abona esta tesis un reciente y novedoso pronunciamiento en la jurisprudencia chilena, en Rosas Bahamondes con Servicio de Salud de Chiloé 2016(19). Este caso trata de una paciente que, en marzo de 2010, se sometió a un procedimiento de esterilización (salpingectomía bilateral mediante técnica de Parkland, o Pomeroy Modificada y una cauterización de cabos de las trompas de Falopio), conjuntamente con una cesárea planificada para el nacimiento de su tercer hijo en gestación. El motivo por el cual se sometió a esta intervención adicional fue que ya era madre de un hijo afectado de hidrocefalia (braquicefalia no sindromática), lo cual, a esa fecha, ya significaba importantes costos de cuidados médicos. Con todo, los profesionales del Hospital de Ancud negligentemente no realizaron la intervención de esterilización agendada, cuestión desconocida por la demandante hasta la comprobación de un tercer embarazo.

Sobre estos argumentos fácticos, la mujer demandó la indemnización de $\$ 1.000 .000$ por los gastos médicos del embarazo, y $\$ 100.000 .000$ 
por concepto de daño moral sufrido por el dolor, la angustia, el sufrimiento y padecimientos sufridos por la demandada causados por la verificación del resultado que la cirugía pretendía evitar.

A la luz de las probanzas rendidas, el tribunal de primera instancia rechazó la indemnización del daño patrimonial, no obstante acogió la indemnización del daño moral, por el monto de $\$ 15.000 .000$. Lo relevante de esta decisión, en lo atingente a esta investigación, es la fundamentación de dicho ítem indemnizatorio. En efecto, el sentenciador determinó que el fundamento preciso de la indemnización se encuentra en la violación del derecho a decidir sobre el propio cuerpo, cuyo sustento normativo, de acuerdo a lo razonado en la sentencia, se encontraría en el artículo $19 \mathrm{~N}^{\circ} 1$ de la Constitución Política de la República, la Convención de Belem do Pará y la Convención sobre la Eliminación de Todas Formas de Discriminación Contra la Mujer CEDAW (considerando 37).

Apelada la sentencia, la Ilustrísima Corte de Apelaciones de Puerto Montt confirmó la resolución con declaración de aumentar la indemnización concedida a título de daño moral a $\$ 35.000 .000$, decisión jurisdiccional que en último término quedó firme.

\section{Ventajas de la fundamentación de las acciones de wrongful conception en la lesión de los de- rechos sexuales de la madre}

Fundamentar las acciones de wrongful conception en la afectación de la libertad de planificar la maternidad permite sofisticar la pregunta inicial. La relevancia de los consensos culturales en cuanto a la vida del que está por nacer(20), permite tomar en cuenta el aparente consenso en cuanto a que es "de sentido común" que el nacimiento de un hijo o hija produce gratificaciones imposibles de valorar económicamente. A su vez, desde la perspectiva del Derecho, es innegable el valor que la vida tiene en el ordenamiento jurídico, a la luz de la regulación constitucional (artículo $19 \mathrm{~N}^{\circ}$ 1) y legal (por ejemplo, artículo 391 del Código Penal, que tipifica el delito de homicidio). Con todo, cabe problematizar, ¿se sigue de esta afirmación de la vida como un valor jurídicamente relevante la inadmisibilidad de estas acciones?
Ante todo, debe tomarse en cuenta que, prácticamente sin excepción, la jurisprudencia nacional y comparada concede la indemnización de los gastos en los que incurren los progenitores por la concepción, embarazo y parto que pretendía evitar sometiéndose a tratamientos anticonceptivos y de esterilización. Luego, lo que parece ser problemático en la jurisprudencia no parece ser la afirmación del valor de la vida humana, sino más bien la cuestión, estrictamente jurídica, de si es razonable que la carga económica de criar a un hijo o hija puede ser impuesta a un tercero.

No obsta a esta argumentación los "beneficios" que representa el nacimiento de un hijo o hija para sus progenitores, pues ellos no son suficiente razón para neutralizar, en términos valorativos y jurídicos, la "invasión a la integridad corporal" de una mujer que, precisamente, quería evitar el embarazo(13).

En el plano jurídico, la apertura conceptual hacia la relevancia de los derechos reproductivos de la mujer tiene importantes consecuencias. Este hilo argumental disuelve la tensión que hemos identificado como transversal en la jurisprudencia chilena y comparada, pues además de la indemnización de los gastos del embarazo y el parto (ya reconocidas por la jurisprudencia chilena), también permite dar un paso hacia la indemnización de algunos gastos asociados con la crianza de un menor. Lo anterior por cuanto permite al Derecho construir razones para admitir que, antes que tratarse de la imposición judicial del derecho de alimentos a personas que no están obligadas a ello, se trata de un dańo (en sentido jurídico) patrimonial de la madre, conceptualmente desligado del derecho de alimentos.

\section{Conclusiones}

Las denominadas acciones por anticoncepción fallida están adquiriendo presencia en la jurisprudencia de los tribunales chilenos. Los casos cubiertos por esta denominación deben ser separados conceptualmente de otros supuestos que se construyen sobre la lesión del interés de los progenitores en interrumpir el embarazo cuando el hijo viene con alguna discapacidad, y esta circunstancia no es advertida por el médico. 
Más allá del problema de la fundamentación que aquí se ha tratado, el tratamiento de este tema abre muchas otras interrogantes para el Derecho, de evidente sustrato valorativo. Así, por ejemplo, podríamos preguntarnos si el padre del hijo inesperado podría demandar por sí mismo la responsabilidad civil en estos casos, o si la discapacidad de la madre es relevante para la determinación del monto de la indemnización. Por otro lado, habrá que preguntarse seriamente si procederá la reparación de los costos de manutención o de crianza del hijo inesperado puesto que, en principio, no se ven razones fuertes para negar absolutamente esta posibilidad. De admitirse esta posibilidad, ¿cómo debería realizarse su pago? ¿A través de una suma única? ¿En mensualidades? Y las alegrías y satisfacciones que reporta tener un hijo, ¿deberían ser tomadas en cuenta para atenuar la indemnización?
Ante nosotros se revela un amplio campo de estudio, comprensivo de cuestiones que tienen el potencial para afectar significativamente la forma en que comprendemos la relación médico paciente y el mercado de prestación de servicios médicos en el ámbito de la reproducción humana. Con todo, el tratamiento de esta cuestión presupone un juicio reflexivo que pondere todos los intereses en juego, y un trabajo interdisciplinario en el que no pueden faltar desarrollos cultivados bajo el alero de las Ciencias Médicas, la Bioética y el Derecho. 
Acciones de wrongful conception en Chile: una propuesta de fundamentación - Hugo Cárdenas, José Antonio Sánchez

\section{Referencias}

1. Jackson A. Action for Wrongful Life, Wrongful Pregnancy, and Wrongful Birth in the United States and England. Loyola of Los Angeles International and Comparative Law Review 1995; 17: 583. Disponible en: http://digitalcommons.lmu.edu/ilr/vol17/iss3/3. [Consultado el 10 de septiembre de 2017].

2. Casals M, Solé J. Anticoncepciones fallidas e hijos no previstos. Dret 2001; 3: 2. Disponible en www.raco.cat/ [consultado el 15 de junio de 2016].

3. Normas nacionales sobre regulación de la fertilidad 2014. Disponible en http://web.minsal.cl/sites/default/files/files/27\%2005\%202014\%20\%20NRF\%20edici\%C3\%B3n\%202014\%20FINAL\%202.pdf. [Consultado el 10 de agosto de 2017]. p. 16.

4. El aumento de la litigiosidad se manifiesta, en términos generales, en las cifras de procesos de mediación obligatoria respecto al sector público como el sector privado (Disponible en http://www.supersalud.gob.cl/documentacion/569/articles-12879_ recurso_1.pdf. [Consultado el 8 de agosto de 2017]).

5. Tironi E. La sociedad impaciente. Los cambios de los chilenos y la salud. En: Chomalí M, Mañalich J, editores. La desconfianza de los impacientes. Reflexiones sobre el cuidado médico y la gestión de riesgos en las instituciones de salud. Santiago, Chile: Editorial Mediterráneo Ltda.; 2006: 19.

6. Casals M, Solé J. Anticoncepciones fallidas e hijos no previstos. Dret 2001; 3: 3. Disponible en www.raco.cat/ [consultado el 15 de junio de 2016].

7. Cfr. Mondaca A, Aedo C y Coleman L. Panorama comparado del wrongful life, wrongful birth y wrongful conception. Su posible aplicación en el Derecho chileno. Revista Ius et Praxis 2014; 21(1): 21.

8. Macía A. Panorama de la responsabilidad civil de los profesionales sanitarios por wrongful birth y wrongful life. Revista Chilena de Derecho Privado 2009; 12: 4. Disponible en http://www.scielo.cl/scielo.php?script=sci_arttext\&pid $=$ S0718-80722009000100005. [Consultado el 15 de junio de 2016].

9. Markesinis B, Unberath, H. The German Law of Torts, a comparative treatise. Oregon: Hart Publishing; 2002. p. 192-7.

10. Borghetti J. France. En: Winiger B, Koziol H, Koch B, Zimmermann, R, editores. Digest of European Tort Law. Berlin: De Gruyter \& Co.; 2011: 880-881.

11. STC 4934/2004.

12. STC $2494 / 2015$.

13. Elvin J. Are Healthy Children Always a Blessing? The Cambridge Law Journal 2002; 61(3): 517.

14. Markesinis B, Unberath H. The German Law of Torts, a comparative treatise. Oregon: Hart Publishing; 2002: 196-197.

15. Corral H. Aborto y esterilización fallidos: ¡el hijo como daño? 27 de mayo de 2012 [Consultado el 25 de agosto de 2017]. En: El blog de Hernán Corral [Internet]. Santiago. Disponible en https://corraltalciani.wordpress. com/2012/05/27/aborto-y-esterilizacion-fallidos-el-hijo-como-dano/.

16. Corte Ap. de Antofagasta, Rol No 373-2011. Corte Ap. de Valparaíso, 3 de julio de 2014, Rol No 2023-2013, LP CL/ JUR/4096/2014. Corte Suprema, 29 de enero de 2016, Rol N 13.544-2015, LP CL/JUR/698/2016.

17. Corte Ap. de Valparaíso, 3 de julio de 2014, Rol No 2023-2013, LP CL/JUR/4096/2014.

18. Facio A. Los derechos reproductivos son derechos humanos. San José: Instituto Interamericano de Derechos Humanos; 2008.

19. Corte Suprema, 29 de enero de 2016, Rol N 13.544-2015, LP CL/JUR/698/2016.

20. Morán JM. La valoración de la vida, la subjetivación del embrión y el debate sobre el aborto: aportes desde una perspectiva crítica. Acta Bioethica 2014; 20(2): 151-157.

Recibido: 9 de diciembre de 2017

Aceptado: 13 de enero de 2018 